DOI: 10.2478/ausfm-2014-0029

\title{
An Olfactory Cinema: Smelling Perfume
}

\author{
Sim Jiaying \\ Nanyang Technological University (Singapore) \\ E-mail: jsim2@e.ntu.edu.sg
}

\begin{abstract}
While technological improvements from the era of silent movies to that of sound cinema have altered and continued to affect audience's cinematic experiences, the question is not so much how technology has increased possibility of a sensory response to cinema, rather, it is one that exposes how such technological changes only underscore the participation of our senses and the body in one's experience of watching film, highlighting the inherently sensorial nature of the cinematic experience. This paper aims to address the above question through an olfactory cinema, by close analysis of Perfume: The Story of a Murderer (2006) by Tom Tykwer. What is an olfactory cinema, and how can such an approach better our understanding of sensorial aspects found within a cinema that ostensibly favours audio-visual senses? What can we benefit from an olfactory cinema? Perhaps, it is through an olfactory cinema that one may begin to embrace the sensual quality of cinema that has been overshadowed by the naturalized ways of experiencing films solely with our eyes and ears, so much so that we desensitize ourselves to the role our senses play in cinematic experiences altogether.
\end{abstract}

Keywords: olfactory cinema, haptic images, sensorial experience in cinema, Perfume: The Story of a Murderer.

What is an olfactory cinema? An olfactory cinema brings to mind a 4-Dimensional cinema, where 3-Dimensional films are accompanied by external technological enhancements that produce additional physical effects, such as gusts of wind, scents, and vibrations. To be clear, this is not the olfactory cinema I wish to consider. While it is true that cinema has yet to come to a point of technological advancement which facilitates an accurate reproduction of smell in cinema, the question is not so much the reasons why smells - as a communicative device fail to make a physical impact in cinema the ways visual and audio media do. In fact, my contention lies within the apparent failures of technological attempts at recreating smells in cinema to suggest that smell is not, and never has been, lacking in cinema. Perhaps, it is best to redirect my question: how is olfaction possible within a cinema that remains ostensibly audio and visual? This demands 
that we approach cinema not just as an exclusively visual and audio medium. Instead, to consider the possibility of cinema as multi-sensory, in spite of this, just like our lived experiences.

I demonstrate an olfactory cinema through Perfume: The Story of a Murderer (Tom Tywker, 2006), which narrates the tale of an orphan named Jean-Baptiste Grenouille, who has a super-heightened sense of smell yet no bodily scent of his own. He ends up murdering young women for their individual scents in order to create the world's best perfume. The establishing shots of Tom Tywker's Perfume: The Story of a Murderer sets up the premise for an olfactory cinema and its implications. We get a montage of quick flashing images that include maggots feasting on decomposing flesh, a wild dog chewing viciously on whatever scraps of meat it can find, rats crawling in and out of butchered meat, the process of gutting fishes and removing innards from pigs; a close up of what seems to be intestines; a man in soiled clothes vomiting by the alley, and a close up of the endproduct [Fig. 1]. This quick montage is juxtaposed to an aerial shot of a bloodied new born baby lying in a pile of dead fish, coupled with amplified sounds of sloshing, butchering, scampering of rats, and vomiting [Fig. 2]. The sensorial and synaesthetic nature of the film images and audio-tracks invites one to experience the film with all our senses, emphasising cinema's olfactory qualities. Although one does not necessarily smell the environment and is unfamiliar with how such a scene should smell, the spectator already experiences the discomfort, senses the dampness of the environment and the stench of the city.

This entire sequence establishes the olfactory landscape of stench, which lasts less than 30 seconds. Although one does not necessarily smell the environment and is unfamiliar with how such a scene should smell, the audio and visual montage is enough for the spectator to feel the discomfort, sense the dampness of the environment and the stench of the city. Similarly, a spectator who has been exposed to an environment as shown in the opening sequence of Perfume: The Story of $A$ Murderer is better able to experience and relate to the stench of the environment, hence enhancing his/her cinematic experience. The sounds of scattering rats, resounding audio track of vomiting, on top of the constant cries of the baby, highlight the ways in which an audio-visual montage may complement one another. Sound, being a more proximal sense as compared to sight, draws the spectator closer to the image presented, almost close enough for one to actually smell.

Disconnected from any character's point of view, the camera moves from different imagery of odours quickly and it is also through the juxtaposition and quick montage that the spectator is assaulted by layers of images and audio 
tracks superimposing on one another, shifting from one to another. The effect of this scene on the spectator is a strong sense of claustrophobia. Such a sequence thus invites the synaesthetic experience of smell and at the same time induces nauseated reactions from this spectacle. While one may take the opportunity to jump in on the fact that this highlights and emphasises the imperatively audio and visual aspects of cinema, I contend that one should resist attributing the impact of this sequence solely to what we see and hear, because only when one makes a conscious effort to break down the sequence can one distinguish the exact visual and audio cues that are presented on screen. In doing so the cinematic spectacle of the sequence is also completely broken down. This synergy - synesthetic, synthesised energy - proposes a multi-sensorial cinematic experience where the senses skirt through their presumed boundaries and overflow into and out of one another, making it difficult to properly separate and identify the senses at work.

According to Carl Plantinga in Moving spectators: American Film and the Spectator's Experience (2009), there are two types of synaesthesia - strong and weak. Only one out of every 2000 persons experiences strong synaesthesia, "a form of cross-modal fittingness," whereby "a subject, words or sounds are accompanied by vivid images of colors or shapes" in the mind of the subject (Plantinga 2009, 157). While the above definitions refer to synaesthesia as clinical condition which only affects a few people, most people experience weak synaesthesia where "the stimulation of one sense cause[s] a perception in another" (Beugnet 2007, 73). This opens up the realm of olfactory, thought to be out of reach in cinema. In this paper, I refer to the phenomenon of weak synaesthesia and suggest that through sounds and visual images in cinema, the sense perception of smell is evoked and one is reminded of how it might be like to smell something. In this way, synaesthesia opens up the realm of olfactory which was thought to be out of reach in cinema.

In fact, scholars Steven Connor and Brian Massumi write about synaesthesia not as "an artistic device, a metaphor, an historical trend, nor solely a rare clinical condition," but "as a way of being in space and time that is simultaneously abstract and very real" (Barker 2008, 237). Through synaesthesia, the boundaries between different senses are blurred since the visual image may evoke the sense of smell. In other words, synaesthesia allows the spectator to be in the particular moment of a cinematic spectacle without necessarily having to experience something physically. One might claim that literal synaesthesia is not possible since cinema is still ultimately audio-visual. However, as Jennifer Barker asserts in her article, Out of Sync, Out of Sight: Synaesthesia and Film Spectacle, it is arguable that the 
opposite is true, that "all cinema is synaesthetic, because in lived experience the senses have a way of skirting the hierarchies and divisions we use to define and explain them" (2008, 237). Since the experience of any sensation is never done so in complete isolation and the boundaries separating different senses are never completely distinguishable, it allows the possibility of synaesthesia in cinema. While smell might have been our first distant sense experience, all the sense modalities have essentially overlapped and have created a commonness in the senses. This underscores the ways which senses are interconnected, facilitating a synaesthetic experience that activates the memory of all other senses, thus inviting the sense of smell even if cinema only engages two senses - sight and hearing.

Therefore, without extradiegetic elements such as actual smells, winds, or splashes of water, cinema is able to "evoke sense experience through intersensory links: sounds may evoke textures; sights may evoke smells (rising steam or smoke evokes smells of fire, incense, or cooking)" (Marks 2000, 213). Its meaning for cinema is straightforward: to pay attention to the sense of smells within a film, spectators can and should go beyond the gimmicks of Smell-O-Vision, or scratchn-sniff cards. Just as the era of silent films led to a cinema which "endow[ed] human beings with a new synaesthetic awareness: spectators will hear with their eyes" (Stam 2000, 35). The very absence of smell should allow spectators to smell with their eyes and ears, as well. While it may be tempting to base the plausibility of an olfactory cinema solely on one's ability to accurately experience the physicality of smells, I argue on the contrary that it is this inability to physically smell in films which welcomes an effective olfactory response to cinema. With that in mind, detractors who are quick to point out the inability for one to 'smell' in a film should realize that it does not matter if one is able to smell physical and actual smells in a film to discourse the experience of olfaction.

In fact, Laura Marks highlights that the physicality of actual smells may not be as successful as an imaginary smell evoked through cinema's audio-visual medium. An actual smell invokes in the spectator separate mental narratives, this 'imaginary signifier' that is the audio-visual of a film enables the spectator to be drawn in as well as out of the spectacle, thus creating a much more profitable interaction between film and the spectator. Marks highlights the evocative nature of an actual smell may distract the spectator from the cinematic spectacle because it evokes another set of narrative within our minds. By experiencing a sense of smell synaesthetically, one is still able to call upon personal experiences through memory, allowing one to respond personally while still ensuring the ability to experience film in its particularity. In other words, the synaesthetic nature of audio-visual images 
provides an opportunity for one to adequately engage with one's sense of smell and the film at the same time, acknowledging the multiplicity of responses to a film without being overtly distracted by one's individual mental narrative.

That said, a synaesthetic sensory experience that encourages olfaction may be called upon through audiovisual cinematic techniques - cuts and edits, use of sounds, mise-en-scène, camera movements. As Martine Beugnet explains in Cinema and Sensation, "very simple and elaborate operations" such as "framing, camera movement, light and contrast, the grain of the image and the mix of different film stocks, as well as the variations in sound and visual intensities" (2007, 74) may invite an olfactory response to cinema. Anna Powell further asserts in Deleuze and Horror Film that "mise-en-scène, cinematography, sound and movement act in the mind/body/body system to stimulate both sensorymotor responses and thought via affect” $(2005,210)$. As such, both Beugnet's and Powell's contentions suggest an implication that synaesthesia is essential to cinematic affect. Affect is one of the vital ways in which spectators experience cinema, and it is this unique cinematic quality that provides a place of departure to consider an olfactory cinema. Powell further explains the Deleuzian concept of affect as a "neuronal response to external stimulus. Qualitative, not quantitative, it involves the body's power to absorb an external action and react internally" (2005, 210). This suggests that while one is exposed to stimulants from the outside and is unable to objectify or quantify it, one still responds to it - be it through facial expressions, body language, or thought.

The aforementioned example demonstrates a few things about cinema: (1) The predominantly perceived audio-visual cinema has produced and conditioned a generation of critics who have become so focused on the importance of visual and audio nuances in cinema, assessing meanings only via one's sense of sight and hearing, that one discounts the potentiality and the participation of other senses. By recognizing cinema as multi-sensory, we acknowledge cinema's potential to activate the other senses - such as our sense of smell, touch, or taste. (2) There is more to a cinematic image than what meets the eye or ear. As Anna Powell asserts in Deleuze and Horror Film, 'the image is not the empty illusion of 'lack,' but is potent with affect. The spectator responds with visceral immediacy to images rather than gazing at them from a subjective distance" $(2005,17)$. The potentiality of an image lies in its ability to call upon instinctive reactions and sensations which are so proximate that one does not merely examine an image with one's eye. As such, an interactive relationship between spectator and film is established - where the film informs the spectator as much as the spectator informs the film. 
Instead of trying to master the cinematic spectacle from a purely cognitive point of view, the spectator is involved in an ambiguous relationship which blurs the line between subjective and objective experience.

In another scene, young Grenouille brings his head close to a dead rat on the ground in order to better sniff the rat [Fig. 3]. After which, he brings his nose close to a dead rat by its tail as he sniffs it thoroughly while picking up the dead rodent. The close-up of the rat is one which draws the spectator closer to the texture of the rat - the stiff fur of the rodent. Before the camera zooms in further and focuses on the stiff fur, the spectator is almost forced to come closer to the image onscreen, thus literally forcing the sense of sight to shift from a distant sense to that of a proximal sense [Fig. 4]. Eventually, through a series of unclear visuals, the spectator is finally shown a close up of the dead rodent's insides filled with squirming maggots. This draws the spectator closer to better appreciate the multi-sensory nature of this image, even if one ends up cringing in disgust at this moment of cinematic affect: coming as close as Grenouille did with a dead rodent. As Carl Plantinga highlights in his book Moving Spectators: American Film and the Spectator's Experience, such disgust is essentially "invoked through that of taste and smell" $(2009,210)$. Such is the visceral and direct response which invokes disgust in the spectator. The synaesthetic experience of smell acts as a sensory stimulus that affects the spectator. In this way, this stimulus evokes a form of disgust in the spectator by bringing to light the imaginations of smells and tastes through synaesthetic presentations of sights and sounds.

The spectator is affected by this moment when s/he may be seeing what Grenouille smells, or s/he is smelling what s/he is seeing. At this instant, not only are the sensory boundaries between that of sight and smell blurred further as we are pushed to such proximity through the cinematic affect of disgust, the boundaries between cinema and spectator cannot be properly defined. These affects posit moments of ambivalence where there is a constant negotiation between the tensions that come from all directions and encounters within and outside of cinema. As such, the spectator is within the film as much as s/he is outside of it. The body of the spectator and that of the filmic body touch at this moment of affect - but this very instant of contact is one which blurs the boundaries between both bodies, and we are engaged in an instant that permits us to belong/not belong in this cinematic experience simultaneously.

Laura Marks argues in her essay Thinking Multisensory Culture that some images appeal to the sense of touch as much as vision does. While it is true that only actual smells call up precious memories associated with them, a haptic 
image requires memory to bring in other forms of association through the refusal to allow visual abundance of any optical image, making it possible for memories to be evoked. Laura Marks calls a haptic image one which encourages the spectator to move closer to it, allowing one to explore it with all of the senses, including touch, smell, and taste (see Marks 2002, 118). The spectator engages with the spectacle by comparing what he/she sees on screen while remembering the mental images that are called up in his/her mind. In the case of the rat, one need not have smelled a dead rat to know of the disgust that accompanies such. At the same time, haptic images "locate vision in the body," making sight behave more like a proximal sense, like that of touch and smell (Marks 2004, 133). Through this, haptic images enable the synaesthetic experience of smell by welcoming an intersensory and embodied perception.

However, what becomes of an olfactory experience when one is unable to identify the scent because of the ab-sense (absence) of any prior encounter or memory with the object of smell? How do we reconcile smell as an absent sense in cinema, yet acknowledge the fact that smell continues to haunt the spectator within the film despite this? There is a scene towards the end of Perfume: The Story of a Murderer that allows me to address this issue. Jean-Baptiste Grenouille is sent for public physical torture, followed by execution in front of a cheering crowd, all in support of his impending death penalty. As he walks up to the stand, he waves a handkerchief in the air, which was previously doused with the perfume made from essences of the girls he murdered. The entire crowd bends forward, kneeling down with hands raised, as if worshipping him. Through the visual images of people and the soundtrack played in the background, one understands that the beauty of this perfume entrances the crowd. The slightly slow-motioned sequence of the entire mass orgy, and the close-ups of contorted facial expressions, attempt to draw spectators even closer to the awe of JeanBaptiste Grenouille's godly scent. Yet at this very moment, even as the spectator searches within his or her memory to find the most fragrant smell which s/he may remember, s/he is left out from this moment of engagement on screen. His godly perfume remains unknowable to the spectator.

According to Deleuze, smell belongs to special category which he calls "the recollection-image, which is an image that encodes memory” (Marks 2002, 123). He suggests that if we are able to call upon the encoded memory within an image, the image no longer remains merely an image, but becomes a narrative. However, when there is a gap between image and memory and we are unable to connect the image to a memory, Deleuze maintains that an image will remain a fossil image. 
This refers to a meaning that is lost in our own bodies and memories because of the inability to evoke it from the image. Due to the lack of ability to smell what we see onscreen and the failure of the image to call forth a memory that might generate a narrative, such desire invoked by the perfume is lost on us.

When one is unable to remember, "the image we perceive does not link up with other images and we perceive it for its own sake" (Trifonova 2010, 144). At this moment, while we are not able to identify with the appreciation or the signification of the scent, we understand the significance of it within the scene. The notion of smell as "significance" and not "signification" is emphasised as the spectator knows of the importance of smell and its effects, despite having its exact meaning escape us. One is able to understand and not understand JeanBaptiste Grenouille at the same time. This is especially true in his case - as even if we could smell, we could never smell like he does, and so the significance of such a scent is always beyond us: his drives are exterior to our capabilities of knowing. For the very knowledge of that would reduce Grenouille's intentions to something that is knowable to us.

This is a very paradoxical situation. By paying attention to smells or the lack of it, we open up an understanding of the film through its own particularity. We withhold moral judgement on Grenouille, who has just murdered young women for the creation of this particular perfume, and attempt to respond to Grenouille as an individual, free from moral rights and law, and begin to empathise with him. This happens even if this means acknowledging our inability to know Grenouille and his motivations completely despite cinematic conventions where one is supposed to relate to and identify with Grenouille through a closeup of his face as he closes his eyes to the flashback of the very scene before murdering the young lady, before opening them to stare briefly and directly at the spectator). The spectator is placed in a precarious position, being affected by different cinematic spectacles of conflicting motives - the juxtaposition of scenes of animalistic desire and coitus to the very sensual and inexplicable desire for a lady or her scent. From this scene alone, I have demonstrated the very way in which an olfactory cinema may allow for the plurality of a film to be explored.

Despite the unknowability of the scent, the "significance" of the scent is understood by the spectator, and attention is still drawn to the ambiguity of this scene as the spectator contemplates their moral position within the film. Therefore, such ambiguity welcomes the film to be read in all its multiplicity, and leaves the narrative open to the spectator's subjective interpretations. As Hans J. Rindisbacher asserts in The Smell of Books: A Cultural Historical Study 
of Olfactory Perception in Literature, bad smells have been noted to signify "repulsion, corruption, decay, and ultimately death," while good smells often suggest a link to attraction, creation of bonds, sexuality, birth and life (1995, 290). What I propose an olfactory cinema can do is to essentially question the very conception of such a binary - good/bad - in the first place. The ability for smell to be experienced within cinema works precisely because of the blurring of any boundaries that seem to keep each sense in their particular sensorial realm. Therefore, the boundaries distinguishing between disgust and desire are never drawn out as clearly as binary opposites make them out to be.

In the final scene of Perfume: The Story of a Murderer, we see a dejected Grenouille, led by the olfactory memories of his childhood, walking back to the very fish market where he was born and abandoned. He stops and stares at a group of homeless people sitting around a bonfire. They appear to be unkempt, dirty, and even primitive in their behaviour. Standing at a distance, Grenouille opens up his bottle of heavenly perfume and empties the entire bottle over his head, dousing himself with this godly-scent. This scene highlights the difficulties and tackiness encountered by cinema in trying to portray smell. Grenouille is lit up in a sort of glow through a spotlight that shines on him to demonstrate how he now glows with a certain godliness, which creates a visual cue to the fact that he has now become an "angel." At this point of time, the upward-tilt of the camera calls into question the spectator's participation and responsibility in this sensory affair. The director pulls the audience into Grenouille's position, and we are forced to question how many of us were also searching for this smell that was missing throughout the entire film. How much of us are the same as Grenouille, gruesomely seeking out this perfect scent, or a scent, regardless of all else?

What follows this close-up is a reverse shot - we see one of the women from the group in complete awe of Grenouille's angelic aura, and how her love for him is immediately aroused by this smell. She runs to him and feeds on his shoulder. A crowd grows around him, almost like the way the rats were portrayed to have fed on decomposing meat at the start of the film as Grenouille stands unmoved, allowing himself to be eaten alive by the raving men and women attracted by his godly-scent. The scene transits via an overlapping crossfade from the crowd feasting on Grenouille until all that remains of him is his clothes. A small boy is shown casually walking by at daybreak, stopping to pick the clothes up as a fortunate find to demonstrate the elapsed time.

This entire sequence leaves the spectator in complete shock because the entire process of Grenouille being eaten alive is presented to the spectator as the passing 
of time, fashioned as a matter of fact with no dramatic effects like the director had used in his portrayal of the godly scent and its effects on the people. There is practically silence - with no words from Grenouille or any of the people in the crowd during the two minutes and four seconds, except for the exclamation of “An angel! Oh, I love you!" before Grenoille allows himself to be eaten alive. The words serve almost no function since one is already able to gather from the sound effects and Grenouille's "glow" that there seemed to be some mystical significance to his perfume creation. However, in such a poignant moment where a sense of Grenouille's grief and resignation is heightened, the synaesthetic nature of imagined smells (i.e. the very streets of Paris where Grenouille was born and his godly perfume) creates a level of emphasis that cannot be expressed otherwise verbally or visually.

This ending sequence also underscores the ways in which desire and disgust are so closely intertwined. The most perfect scent in the world could in fact induce such disgust - the things one would do to attain such a perfume, and what eventually happens when the scent is used. The line between disgust and desire is all the more blurred and such closeness of "life, with erotics and sexuality, as its climax, and death" is perhaps best expressed in the French expression, "la petite mort" of which literal translation means "little death" (Rindisbacher 1995, 290). Such an expression for orgasms suggests effectively the closeness between death and desire, or disgust and desire. Perhaps one of the greatest pitfalls of Perfume: The Story of a Murderer is its emphasis on and portrayal of the sense of smell as something "dangerously savage," as suggested by Watson (2000, 112). Grenouille is characterised as a man whose obsession with trying to create the world's perfect scent leads him to stalk and murder women who were not in the know. This is as opposed to drawing on the extraordinary nature of Grenouille's sense of smell, making it to be a true talent or a potential that is rare and barely explored enough. His olfactory sense allows himself to learn quickly the art of perfumery, as he is able to create scents without having to abide by actual proportions.

As the film demonstrates, the sense of smell, like any other sense, is one which has to be cultivated as well. We see Grenouille growing up, curious about every single smell sensation around him as he walks around linking each scent to its object, building his own olfactory vocabulary and library. However, the film subverts the possible potentialities of such an olfactory talent in various ways. The narrator whose voice appears throughout the film recounts the growth of Grenouille as he discovers his hidden nose for beautiful scents, subverts the positivity of such a "talent" by having to spell out and articulate with words that it is in fact a talent, 
as if one is unable to notice that for themselves. Also, a parallel is drawn between Grenouille's lack of personal odour and his apparent lack of a moral conscience which causes him to murder without guilt. However, through an olfactory cinema, one is able to subvert the superficial presentations of his suggested talent, built up through audiovisual images, to expose a deep-seated layer of distrust towards the film with regards to the idea of such a sensory prowess.

Simply by setting up the premise for an olfactory cinema, one finds that skepticism towards adopting such an approach encompasses something deeper and more complex than just debates about technological apparatus, or the incommunicable nature of olfaction. Since synaesthesia allows the sense of smell to be evoked, apparent concerns serve more as an excuse and an attempt to conceal what lies at the crux of such an apprehension towards an olfactory cinema - the general distrust towards our sense of smell. Such skepticism towards the sense of smell is unsurprising considering how smells have remained elusive to us. We often find it difficult to articulate smell experiences without utilizing another sensory medium, or comparing it to something else. Dan Spercer brings up this problem of smells in Rethinking Symbolism, highlighting how our knowledge of different smells is determined by all the "categories whose referents have olfactive qualities," instead of having an independent domain of its own (quoted in Rindisbacher 1995, 15). Language has yet to develop a set of vocabulary for smells as we find ourselves describing smell in relation to its origin, or something else because our inability to "name" a smell as it is.

While one is able to acknowledge such an olfactory experience, one is unable to effectively put a cognitive encoding to the exact smell that one is experiencing without a relation to another. For example, the "smell of roses," or "it smells like roses" highlight the very metaphorical nature of smells. Due to such difficulties in articulation, smells have become "factually eliminated, linguistically euphemised, [and] psychologically repressed" (Rindisbacher 1995, 8). However, to avoid the address of smell altogether simply because of an inability to properly communicate it would be to suggest that significance lies only in how much something may be translated into common language.

Furthermore, as Anna Powell rightly puts in her book Deleuze, Altered States and Film (2007), such "immediacy of direct apprehension bypasses the capacity of words to fix phenomenal meaning" $(2007,101)$. This suggests that smell avoids being reduced to a single phenomenon, which ignores the multiplicity of any encounter. The sense of smell thus effectively exemplifies how one is not always able to translate the world into a common language or experience which is relatable 
to all. Hence, the inability of smells to be properly communicated linguistically does not equate to its insignificance. Similarly, a physical absence of smells in cinema does not mean that olfaction is unable to affect our cinematic experience.

However, as Laura Marks states in The Skin of the Film, the use of senses are "variable according to culture and local need" $(2000,202)$. As such, our usage of senses is akin to a skill which one has to pick up. We learn more about any sense through regular usage of it and the use of senses differs from one culture to another. Thus, this suggestion echoes Norbert Elias's take on the importance of sight only in today's society, "the eye takes on a very specific significance in civilized society" $(1994,203)$. Robert Mandrou also asserts that despite the relative unimportance of the sense of smell and taste as compared to the other three senses in modern day, "the men of the sixteenth century were extremely susceptible to scents and perfumes" (quoted in Jenner 2000, 130). This suggests that every culture differs in its use of the senses and exposes how our biases against the sense of smell may be a product of social construction, so much so that the disregard for smell has become accepted as a natural belief.

As society turns toward modernity, the sense of smell decreases in importance while the sense of sight gains significance. Under such conception, I reiterate my point that the sense of smell is as valuable as any of the other senses, but it has been eradicated as a sense that remains below the hierarchy of sight and hearing through unquestioned social construct. Thus, any hesitance towards the usefulness of an olfactory cinema implies a strong inclination to unthinkingly accept social norms that have been based on nothing more than biases that have gone unquestioned. By opening up the possibility of such an approach to cinema, one is already making an attempt to embrace smell in all its materiality and value in its own right - the ability for it to open up the plurality within a film. That in itself is an act to refute totalizing analysis made through primarily audio-visual responses.

At this point, I wish to assert that my examples are singular by nature and they are only a demonstration of how I may apply an olfactory approach to cinema. They are by no means a definitive response to the films. What one takes away from an olfactory approach to cinema may and would differ from person to person as one takes into account the many differences that would shape one's encounter with cinema. This is the reason why an olfactory cinema better facilitates the exploration of multiple potentialities within a film. One would suspect that even without the introduction of gimmicks and technological experiments, one would be wary of any attempts to portray the very elusive, and intangible sense of smell through audio-visual means. Thus, to open ourselves to the very idea that smell 
has all along been inherent within films, regardless of its physicality in cinema, may prove disconcerting for some.

Given the ways smell has already been neglected as one of the least important senses as compared to our senses of sight and hearing, per se, one cannot help but wonder how much we actually do know about smells and how much one can draw from an olfactory approach to cinema. However, what we do know are the potentialities and the values that an olfactory cinema affords; the film posits a certain level of dynamism over the film because of the ways olfactory allows an interaction between film and spectator. In this way, this relationality creates meaning that is singular and unique to each individual's cinematic experience. Different approaches to cinema open up various frameworks of responses to cinema, each defending their own approaches as beneficial to the cinematic experiences. However, the concern is not so much which approach to cinema is most beneficial and effective. There is no ranking in the ways of experiencing the world, or cinema; only different ways of approaching and responding.

I am offering only a prolegomenon to a larger investigation - the possibility of exploring even more approaches that could shed light on our understanding and experience of cinema. By opening up this other paradigm in cinema, spectators are equipped with new ways of understanding what was previously left out through a solely audiovisual approach. The nature of spectatorship is one that shifts constantly with various factors - the text, apparatus, history, social contexts, where spectators are involved in an ever-changing conversation with the cinematic experience. One must take into consideration the possibility of a future that could welcome the accurate experience of smell in new technological innovations in apparatus, one which would alter once again our olfactory experience in cinema. Or perhaps an olfactory approach might influence shifts in filmmakers' intentions to deliberately call upon synaesthetic responses of smell in cinema. Hence, at the very least, an olfactory cinema reminds us of what cinema can do - its ability to open up unexplored areas of experiences and provide a plenitude of meanings. 


\section{References}

Barker, Jennifer. 2008. Out of Sync, Out of Sight: Synaesthesia and Film Spectacle. Paragraph vol. 31 no. 2: 236-251.

Beugnet, Martine. 2007. Cinema and Sensation. Edinburgh: Edinburgh University Press.

Elias, Norbert. 1994. The Civilizing Process. Oxford: Blackwell Publishers.

Jenner, Mark. 2000. Civilization and Deodorization? Smell in Early Modern English Culture. In Civil Histories: Essays Presented to Sir Keith Thomas, eds. Peter Burke, Brian Harrison and Paul Slack, 127-145. Oxford: Oxford University Press.

Marks, Laura. 2000. The Skin of the Film: Intercultural Cinema, Embodiment and the Senses. North Carolina: Duke University Press.

Marks, Laura. 2002. Touch: Sensuous Theory and Multisensory Media. Minneapolis: University of Minnesota Press.

Marks, Laura. 2004. Thinking Multisensory Culture. Paragraph vol. 31 no. 2: 123-137. Edinburgh: Edinburgh University Press.

Plantinga, Carl. 2009. Moving spectators: American Film and the Spectator's Experience. London: California University Press.

Powell, Anna. 2005. Deleuze and Horror Film. Edinburgh: Edinburgh University Press.

Powell, Anna. 2007. Deleuze, Altered states and Film. Edinburgh: Edinburgh University Press.

Rindisbacher, Hans J. 1995. The Smell of Books: A Cultural-Historical Study of Olfactory Perception in Literature. USA: Michigan University Press.

Stam, Robert. 2000. Film Theory. An Introduction. Malden MA, Oxford: Blackwell Publishing.

Trifonova, Temenuga. 2010. A Nonhuman Eye: Deleuze on Cinema. SubStance vol. 33 no. 2 (2004): 134-152.

Watson, Lyall. 2000. Jacobson's Organ and the remarkable nature of smell. London: Penguin Press. 


\section{List of Figures}

Figures 1-2. Maggots on a rotting pile of dead fish seen in the opening montage: Perfume: The Story of A Murderer (Tom Tykwer, 2006). Newborn Jean-Baptiste Grenouille crying after being assaulted by the smells at the fish market.
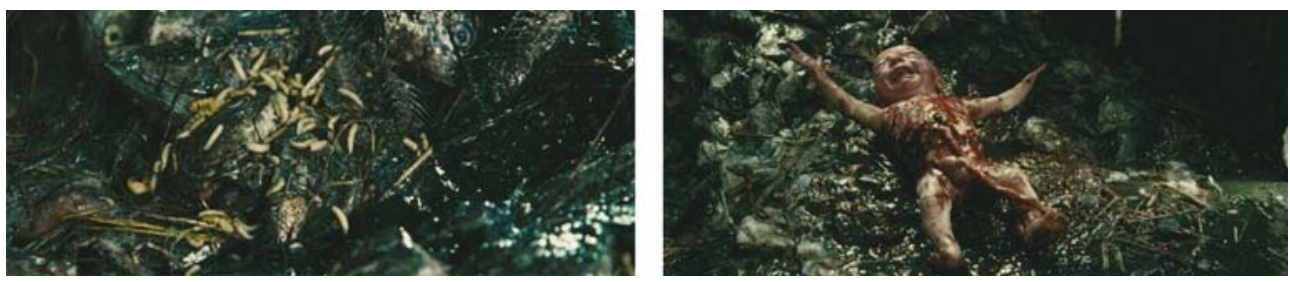

Figures 3-4. Young Grenouille smelling a dead rat. Close-up of the dead rat's stiff fur.
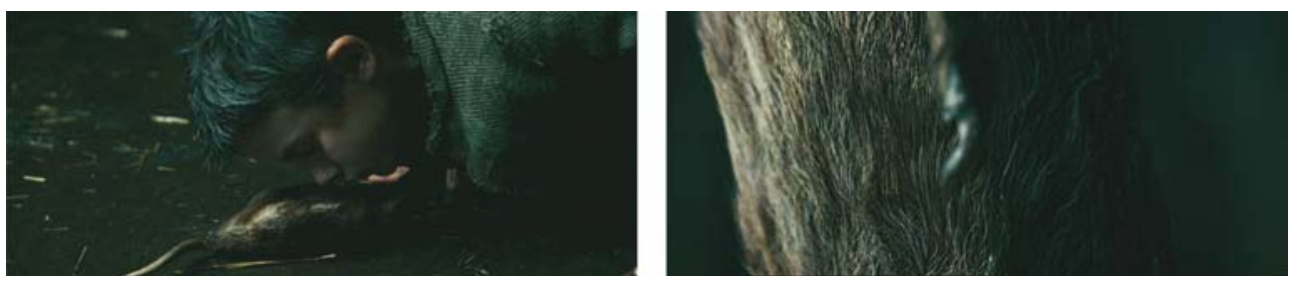